\title{
I NUOVI SINDACI: COME CAMBIA UNA CARRIERA POLITICA
}

\author{
di Gianfranco Bettin Lattes e Annick Magnier
}

\section{La carriera del sindaco nella ricerca pionieristica}

Il termine carriera, qualunque sia il campo concreto di riferimento, unisce un elemento dinamico - usualmente percepito con una valenza ascendente - con un elemento tendenzialmente stabile, riconducibile ad un progetto prefigurato in un dato ambito istituzionale. A comprovare l'ambiguità semantica del termine stanno due suoi caratteri antitetici solo in apparenza. In primo luogo, l'aspetto istituzionale storicamente consolidato nei termini di un percorso potenziale a disposizione degli attori dotati ed interessati; in questo senso, la carriera è lo schema organizzativo d'una professione ordinata ${ }^{1}$. In secondo luogo, l'aspetto soggettivo connesso all'attore che intraprende, svolge e matura la sua carriera. Questo secondo aspetto, non certo secondario sotto il profilo sociologico, introduce un elemento di alea legato all'adempimento effettivo della carriera, la quale in quasi tutti gli ambiti istituzionali non prevede uno svolgimento completo in una forma rigida. La definizione della carriera dipende dalle modalità della sua interpretazione soggettiva in una misura che sembra assai interessante studiare empiricamente.

Che anche i politici siano titolari di una professione e che

Questo saggio si collega ad un'esperienza di ricerca su «La sociologia del potere nella città italiana contemporanea», finanziata con contributo CNR n.90.0256410, in via di completamento.

${ }^{1}$ La riflessione sulla nozione di carriera ha visto un suo momento significativo nei lavori della seconda generazione della Scuola di Chicago, focalizzati sullo studio delle professioni prima e sulla definizione della carriera dell'attore deviante, poi. Tra i tentativi di definizione e di tipologia utili nella prospettiva qui adottata, meritano di essere citati E.C. Hughes (1951), H.S. Becker (1963) e J.R. Tréanton (1960). Nell'ambito più pertinente alla scienza politica resta fondamentale il contributo di D. Herzog (1975) e, più recentemente, quello di $\mathrm{A}$. King (1981).

RIVISTA ITALIANA DI SCIENZA POLITICA / a. XXV, n. 1, aprile 1995 
come tali abbiano da percorrere una loro carriera sembra essere un dato acquisito sia nella mentalità del cittadino comune sia nella mentalità dello studioso addetto ai lavori ${ }^{2}$. Una simile consapevolezza, tuttavia, è un'acquisizione relativamente recente, come risulta dalle testimonianze di autori, da Weber a Michels a Schumpeter, per citare solo i classici, che hanno insistito su questo dato per evidenziarne le implicazioni non univoche. L'idea della politica come servizio svolto a beneficio della comunità, quindi come attività occasionale che non diventa mai una vera professione, rimane comunque un punto di riferimento altrettanto diffuso sia nella cittadinanza che nella letteratura specialistica. È inaccettabile la tesi di coloro che sostengono che una carriera politica in senso proprio si intraprende e si compie unicamente al livello nazionale mentre alla periferia del sistema politico allignerebbero politici di second'ordine titolari di un'esperienza poco significativa. Le cose non stanno così: il rapporto tra la carriera politica periferica e la carriera che si può maturare al centro è per molti aspetti un rapporto diretto e caratterizzato da reciprocità fra i titolari dei vari ruoli di potere. La reciprocità diventa operante sotto molteplici profili, nel senso che chi sta al centro del sistema politico costruisce la sua posizione controllando una serie di posizioni periferiche e numerose altre carriere: far politica significa, oggi come ieri, lavorare con risorse che determinano chances di mobilità nell'area della politica e in territori circumvicini. L'elemento «controllo politico delle carriere» assume un ulteriore peso quando si entri in concorrenza con altri politici per avanzare nel cursus honorum, nell'ovvio senso che le possibilità di carriera vengono a dipendere più che mai dall'intreccio delle alleanze giuste, specialmente con chi sta al centro del sistema.

Cercheremo, in questo articolo, di verificare quali trasformazioni questa carriera ha subìto nell'ambito del nostro sistema politico ed, infine, che senso ha - se ce l'ha - parlare di una carriera al livello del sistema politico periferico e, segnatamente, al livello specifico della carica di sindaco di una città italiana una posizione che si può ritenere probabile punto di arrivo, coronamento definitivo e premiante di un iter politico per l'ap-

${ }^{2}$ La letteratura sul professionismo politico si sta incrementando in questi ultimi lustri, pur tuttavia con un apporto di ricerche empiriche assai limitato. Sul tema si rinvia, tra gli altri, a G.S. Black (1970 e 1972), P. Farneti (1972), G. Sani (1972), D. Gaxie (1973), R.D. Putnam (1976), M. Cotta (1979), A. Mastropaolo (1984 e 1993). 
punto periferico. Il caso del sindaco appare emblematico per più motivi. Indipendentemente dai contesti ambientali e dalla dimensione del comune, il ruolo di primo cittadino mantiene una sua unità e si qualifica come un segmento significativo delle carriere esperite nell'ambito del sistema politico italiano. La prima osservazione da avanzare è che tra tutti i ruoli definiti alla periferia del sistema politico, quello del sindaco sembra offrire le maggiori opportunità per uno stile di leadership che riflette la tendenza alla personalizzazione nella gestione del pote$\mathrm{re}^{3}$. Tanto più oggi, dopo le prime applicazioni della legge 81 del 25 marzo 1993 sull' «Elezione diretta del sindaco, del presidente della provincia, del consiglio comunale e del consiglio provinciale», che ha rafforzato in chiave monocratica il ruolo del primo cittadino, sia a monte con le procedure di elezione diretta, sia a valle con la definizione delle sue prerogative di capo del governo della macchina municipale. Nella mappa complessiva del potere locale, la posizione di sindaco appare come un traguardo assai ambito. Lo studio empirico sul momento fondamentale dell'accesso alla carica dimostra il possesso di qualità che illuminano non solo i meccanismi di reclutamento in questa posizione particolare, ma che aiutano a chiarire le dinamiche attive all'interno dell'arena municipale nel suo insieme. Sul sindaco, poi, possediamo alcuni studi di riferimento che permettono di ricostruire, sia pure grossolanamente, uno schema euristico diacronico di medio periodo 4 .

La tipologia più classica, sulla quale si fonda il modello interpretativo di Sidney Tarrow, distingueva il sindaco notabile

${ }^{3}$ Sulla personalizzazione del potere, anzi meglio, sulla «personalizzazione della leadership politica» si vedano i saggi fondamentali di L. Cavalli (1987, 1992 e 1994).

${ }^{4}$ Le ricerche che consentono di ricostruire l'evoluzione del sistema politico italiano per quanto riguarda il reclutamento e l'attività di governo svolta dai sindaci sono, come si sa, assai poche. La prima è stata quella di A. Spreafico (1963). Questa ricerca ha saputo utilizzare le biografie dell'Annuario Politico, redatte in base a un questionario compilato direttamente dagli interessati ed integrate con alcuni dati allora forniti dall'Istituto per il progresso dell'amministrazione pubblica. La seconda ricerca importante è stata quella di S. Tarrow (1979); si trattava di un'indagine comparata fondata su un questionario rivolto a 117 sindaci francesi e a 131 sindaci italiani. Vanno poi ricordate le analisi dell'anagrafe del servizio elettorale del Ministero dell'Interno proposte dall'inizio degli anni Settanta da C. Barberis in successive edizioni (1973, 1978, 1983, 1988, 1993) e le ricerche svolte da G. Martinotti (1988). Per delle utili indicazioni interpretative complementari sui sindaci dei primi anni Sessanta a partire da un insieme di 1346 sindaci inclusi nell'Annuario dei Sindaci d'Italia del 1964, si veda R. Segatori (1992). Più recentemente presenta un indubbio interesse metodologico la tipologia dei sindaci italiani proposta da E. Recchi (1993). 
tradizionale dal sindaco contemporaneo, dotato di minor prestigio familiare e personale nella comunità, le cui risorse di potere politico gli sono principalmente fornite dal partito che lo forma e lo promuove. Va ricordato che già all'inizio degli anni Settanta la figura del sindaco tradizionale era, sempre secondo Tarrow, meno diffusa in Italia che in Francia - anche in ambiente rurale. La stessa distinzione che storicizza gli elementi definitori del ruolo del primo cittadino, sembra di non poca utilità per una lettura dei dati usati da Alberto Spreafico, nell'ormai lontano 1963, con riferimento al governo dei comuni italiani capoluogo. La politica municipale, al livello del sindaco di capoluogo, appariva, alla pari di quanto avveniva nei centri minori, come un'attività tradizionalmente aperta ai figli di un'aristocrazia locale indipendente nei suoi mezzi di sostentamento e con grandi disponibilità di tempo. Questi sindaci, liberi professionisti, benestanti ed imprenditori, si potevano definire notabili in un senso weberiano. In una fase successiva, a questi si affiancano sindaci che hanno un'estrazione sociale di tutt'altro segno e che vedono nella politica l'occasione per una carriera o, comunque, un'opportunità di mobilità sociale ascendente per sé e per i propri figli. Sembra di potere affermare che soprattutto per chi era di estrazione sociale operaia ed impiegatizia operasse, in termini motivazionali, una concezione ambivalente della politica: da una parte come servizio occasionale prestato in cariche elettive a beneficio della comunità locale, dall'altra come carriera ordinata.

Nel 1963, l'analisi dei dati riportati dall'Annuario politico consentiva, in effetti, una valutazione della posizione professionale dei sindaci al momento dell'elezione ed un confronto con la posizione professionale del padre che illustrava, per i grandi comuni, la persistenza, si tratta di sei lustri or sono, del modello tradizionale del sindaco notabile. I liberi professionisti costituivano allora un terzo del totale, seguiti a breve distanza dagli insegnanti. Venivano poi gli impiegati (il cui settore economico di attività era e rimane dai contorni piuttosto incerti) e i dirigenti, sia pubblici sia privati. La proporzione di sindaci che definivano la loro posizione professionale in modo da apparire come veri e propri «organizzatori politici e sindacali» era molto ridotta, assai minore di quella rilevata tra i deputati alla stessa data. Sempre nell'indagine del 1963, l'esperienza elettiva pregressa, calcolata in termini di tempo impegnato negli organi elettivi comunali, provinciali o regionali appariva spesso ridotta: 
6 anni in media. Il gruppo più consistente di sindaci (circa un terzo) aveva da 2 a 5 anni di esperienza elettiva periferica al momento dell'elezione, un sesto tra 5 e 10 anni. E da rilevare tuttavia che un quinto non aveva nessuna esperienza elettiva periferica: si trattava per la maggior parte di uomini dell'apparato (come dimostra la forte proporzione di eletti ex-comunisti in questa categoria). Le tappe più usuali, se non obbligatorie, della carriera pregressa erano, sempre secondo la diagnosi di Spreafico, quella dell'assessorato comunale o provinciale, quella della delega a vicesindaco o dell'apprendistato come sindaco di un comune minore (ma questa esperienza formativa di passaggio valeva soltanto per un decimo dei sindaci). Da questo rapido quadro empirico dedicato alle carriere dei sindaci si poteva dedurre, già trent'anni fa, l'importanza predominante della preparazione interna alle strutture partitiche rispetto a quella negli organi elettivi del comune. Dagli stessi dati, si può forse inferire anche che il ricorso a personalità non provenienti dagli apparati di partito, notabili o meno, fosse già allora più alto di quanto si potesse credere. Questo tipo di politico sui generis era di norma assai fedele al partito e poteva molto spesso vantare una ragguardevole anzianità di iscrizione; anche se non era inserito nelle trafile usuali della politica periferica e non si era sottoposto ai ritmi burocratici della carriera, aveva conosciuto esperienze personali significative impegnandosi pubblicamente.

La prospettiva metodologica che ha orientato la rilevazione dei dati sui sindaci degli anni Ottanta e Novanta riflette un'impostazione analitica di tipo storico-istituzionale che si preoccupa di disegnare e di verificare delle ipotesi sulle dinamiche di trasformazione del sistema politico periferico nel medio periodo. Questa prospettiva è stata collaudata grazie all'attuazione di un programma pluriennale di indagine focalizzato sull'intero universo del personale eletto membro della classe politica municipale italiana dal 1987 al 1993. Tale impostazione converge con l'interesse diffuso che le nuove generazioni di scienziati politici e di sociologi hanno dimostrato per il tema a partire dagli anni Ottanta $^{5}$. Nella fattispecie, il programma di ricerca sulla classe politica municipale del team fiorentino, nell'ambito di una im-

${ }^{5}$ Per una rassegna critica dei numerosi studi che testimoniano dell'interesse rinnovato per la leadership politica periferica a partire dalla fine degli anni Settanta, sia consentito rinviare a G. Bettin (1993) e alle originali ricerche empiriche di N. Porro (1993) e di V. Belotti, M. Maraffi (1994). 
postazione posizionale, ha tentato di descrivere i mutamenti avvenuti nel sistema politico periferico successivamente alla prima fase di rivalutazione sociologica della dimensione politica locale della fine degli anni Sessanta. Dopo la ricerca su Il consigliere comunale (1989) e quella su Chi governa la città? (1991) dedicata agli assessori dei grandi comuni italiani, il programma si conclude con un ampio lavoro di rilevazione relativo ai primi cittadini in carica nei comuni italiani tra il 1992 e il 1993. Queste date consentono di verificare la reale incidenza di un processo di mutamento politico-istituzionale dipendente dalla legge 81, ma anche dagli effetti di un più ampio processo di delegittimazione di quei partiti che erano fino a ieri la pietra angolare del sistema politico nazionale, sia al centro che in periferia. A un campione di sindaci in carica nel 1992, vale a dire poco dopo l'approvazione della legge 142/90 che promuoveva l'autonomia statutaria ed innovava fortemente nel governo delle municipalità, veniva somministrato un questionario di 113 domande che tendeva alla descrizione analitica dei processi di socializzazione politica, di attivazione, di selezione e di promozione dei primi cittadini. Con lo stesso strumento si è potuto procedere alla rilevazione dei dati relativi alla vita quotidiana dei sindaci (rete relazionale con altri, politici e non; bilancio temporale; modalità di formazione dell'agenda); all'autopercezione del ruolo; ed, infine, alla definizione di alcuni atteggiamenti politici e delle opinioni sulle riforme in corso. L'analisi intensiva delle numerose informazioni raccolte attraverso un campione stratificato di 307 sindaci in carica nel 1992 consente di delimitare meglio un ruolo finora mal descritto nella letteratura scientifica, malgrado la sua importanza crescente nella relazione tra cittadino e sistema politico. La stessa rilevazione è stata ripetuta su tutti i sindaci eletti nelle prime due tornate amministrative successive all'approvazione della legge 81 (6 e 20 giugno 1993, 21 novembre e 5 dicembre 1993). Queste consultazioni elettorali hanno avuto luogo rispettivamente in 1.192 e 424 comuni. Qui di seguito si presentano alcuni dati dichiarati dagli 865 sindaci eletti nel 1993 che hanno partecipato all'indagine rispondendo al questionario. Essi costituiscono più di metà dell'intera popolazione dei nuovi sindaci ${ }^{6}$. L'obiettivo di questo esperimento è quello di controlla-

\footnotetext{
${ }^{6}$ Un confronto con i dati dell' anagrafe del servizio elettorale del Ministero dell'Interno sui sindaci eletti in queste due tornate (con riferimento alle quali, al 15 ottobre
} 
re, sotto l'angolatura specifica del personale politico, i primi cambiamenti concreti introdotti dalla riforma nel funzionamento della democrazia al livello locale.

\section{Il sindaco degli anni Novanta: notabile o «parvenu»?}

La lettura dei dati sociografici può condurre ad esiti più fruttuosi che non un semplice quadro tassonomico riepilogativo di come si distribuiscano alcune variabili caratterizzanti il gruppo dei primi cittadini in carica. Nella prospettiva interpretativa specifica di queste pagine si intende disegnare una tipologia dei pattern di carriera articolabile sulla base di alcune risorse soggettive ed istituzionali tipiche dei sindaci. Gli stessi dati sociografici mostrano che la competizione per il potere, via via che si sale nella gerarchia politica municipale, passa attraverso percorsi socialmente differenziati, correlati ad un insieme di abilità con un'influenza a volte determinante. Ovviamente ogni aspetto, valutato nella direzione di lettura del campo prescelta, invita ad una verifica empirica la più accurata possibile sotto il profilo empirico. Le generalizzazioni presentate in questa sede vanno, tuttavia, accolte con la dovuta cautela perché si privilegia praticamente una sola fonte di dati: l'autorappresentazione che i sindaci fanno di loro stessi tramite le risposte ad un questionario.

Corrispondenze e divergenze tra la composizione sociale della classe politica municipale e le caratteristiche della società italiana sono state più volte analizzate anche nelle loro tendenze storiche di trasformazione ${ }^{7}$. Alla vigilia dell'applicazione della legge 81 , la «legge di rarefazione progressiva», verificata in genere per le cariche elettive al livello municipale, si confermava

1994, risultano registrati nell'anagrafe ministeriale 1540 sindaci), dimostra che il nostro campione non presenta distorsioni significative. In particolare, ciò vale per quanto riguarda le variabili consuete, dalla distribuzione geografica dei sindaci (Nord: $33,44 \%$ nell'anagrafe ministeriale, $38,95 \%$ nel nostro campione; Centro: 11,49 e $11,68 \%$; Sud e isole: 55,07 e $49,36 \%)$, al sesso $(7,30 \%$ di donne nel nostro campione, $6,30 \%$ nell'anagrafe ministeriale). Per quanto riguarda, poi, il titolo di studio, le leggere variazioni e la presenza di campi vuoti nel file del Ministero, lasciano supporre una sovravalutazione di circa l'1\% dei sindaci laureati; anche per quanto riguarda l'età, lo scarto maggiore riguarda le due fasce estreme - dei sindaci con meno di 31 anni e dei sindaci con più di 70 anni - e non supera, comunque, $1{ }^{\prime} 1 \%$.

${ }^{7}$ Sembra inevitabile il riferimento alle ricerche sulla classe politica municipale già citate di C. Barberis e a quelle di G. Bettin e A. Magnier (1989 e 1991). 
valida anche per la carica apicale di primo cittadino ${ }^{8}$. Alcune categorie sociali vengono poco o punto rappresentate nei consigli comunali; la rarefazione si accentua progressivamente mano a mano che si sale nella scala del potere municipale: la legge si verifica in modo forte per le categorie dei giovani, delle donne, degli operai. Solo il 2,3\% dei sindaci in carica nel febbraio 1993 aveva meno di trent'anni; più spazio era concesso all'altra categoria marginale, gli ultrasessantenni, con un quota del $12,2 \%$ dei sindaci. Paragonando queste percentuali con quelle del 1987 (i sindaci sotto i 31 anni erano il 3,7\% e quelli oltre i sessanta l'11,2\%), ci si rende conto di come la rarefazione abbia ulteriormente colpito la prima fascia di età e premiato leggermente l'ultima. Il dato poteva essere interpretato come indicativo di una tendenza ad un maggiore apprezzamento della risorsa «esperienza» in un mondo che invecchia e che innalza l'età d'ingresso dei giovani nell'età adulta, oppure, più cinicamente, come segno di arroccamento e di chiusura di un ceto politico che manteneva saldo il controllo delle cariche di governo a dispetto del proprio invecchiamento fisico. I dati rilevati sui sindaci eletti dopo la legge 81 inclusi nel nostro campione nazionale avvalorano maggiormente la seconda ipotesi. Non si può dire che si sia consolidata nel tempo una tendenza gerontocratica nel sistema politico municipale, in quanto gli ultrasessantenni rappresentano solo 1'8,5\% dei sindaci eletti nelle due consultazioni elettorali di giugno e novembre 1993 (tab. 1). In generale, il cambiamento della classe politica municipale, per effetto della riforma elettorale e delle presenze sulla scena di nuove formazioni politiche, non assume una dimensione generazionale. I nuovi sindaci con meno di 31 anni sono il 3,6\% e registrano un incremento assai tenue rispetto al 2,3\% del febbraio 1993. Ma, nel complesso, i valori riguardanti i nuovi sindaci sono quasi identici ai valori registrati nel 1987.

Si conferma anche ulteriormente la predominanza della fascia di età centrale come principale area demografica di reclutamento dei sindaci. Sia che si faccia riferimento ai dati forniti dall'anagrafe del servizio elettorale del Ministero dell'Interno, sia che si utilizzino le dichiarazioni dei nostri sindaci, tanto nel

\footnotetext{
${ }^{8} \mathrm{I}$ dati analizzati in questo paragrafo provengono dall'anagrafe del Ministero dell'Interno, aggiornata al 15 febbraio 1993 e riguardano i 7.869 comuni registrati, su un totale di 8.049 .
} 
TAB. 1. Età e livello sociale dei nuovi sindaci (\% di colonna)

\begin{tabular}{|c|c|c|c|c|c|c|c|}
\hline \multirow[b]{2}{*}{$\begin{array}{l}\text { LIVELLO } \\
\text { SOCIALE }\end{array}$} & \multicolumn{6}{|c|}{ ETÀ } & \multirow[b]{2}{*}{ Totale } \\
\hline & $21-30$ & $31-40$ & $41-50$ & $51-60$ & $61-70$ & $>70$ & \\
\hline Borghesia & 48,4 & 45,7 & 36,7 & 38,4 & 24,6 & 16,7 & 38,6 \\
\hline $\begin{array}{l}\text { Piccola borg. } \\
\text { dipendente }\end{array}$ & 12,9 & 44,8 & 49,5 & 34,1 & 8,2 & 0,0 & 40,5 \\
\hline $\begin{array}{l}\text { Piccola borg. } \\
\text { indipendente }\end{array}$ & 12,9 & 3,6 & 4,5 & 4,3 & 4,9 & 8,3 & 4,6 \\
\hline Operai & 0,0 & 1,4 & 1,6 & 0,6 & 0,0 & 0,0 & 1,2 \\
\hline Non definito & 25,8 & 4,5 & 7,7 & 22,6 & 62,3 & 75,0 & 15,1 \\
\hline Totale & 3,6 & 25,5 & 43,5 & 19,0 & 7,1 & 1,4 & 100,0 \\
\hline
\end{tabular}

Fonte: Nostro campione: 865 sindaci eletti nel 1993.

1992 quanto nel 1993, la variabile «età» sembra essere preponderante rispetto alla variabile «classe sociale»", nel senso che in ogni strato sociale le maggiori chances di accesso alla carica di sindaco sembrano riservate a chi ha tra $\mathrm{i}$ quarantuno e $\mathrm{i}$ cinquant'anni. L'unica anomalia si registra all'interno dei ceti superiori indipendenti, fra $i$ quali sono sovrarappresentati $i$ trentenni mentre si assiste ad un netto calo di presenze dopo i cinquant'anni. Forse il sindaco proveniente dagli strati più alti era avvantaggiato nel raggiungere anticipatamente un incarico che

\footnotetext{
${ }^{9}$ Per la classificazione della condizione socioprofessionale dei sindaci, il Ministero dell'Interno utilizza, a partire dal 1992, la nomenclatura ISTAT. Nella nostra prospetti$\mathrm{va}$, in alcuni casi, la tassonomia ISTAT appare riduttiva ed è stata corretta (un esempio un po' eterodosso, ma utile: i medici chirurghi sono considerati dall'ISTAT parte dei ceti superiori, mentre $i$ dentisti sono collocati fra $i$ ceti medi). Salvo alcuni spostamenti di singole categorie professionali (nel caso dell'esempio, i dentisti nel ceto superiore), si è tuttavia cercato di rispettare il più possibile la classificazione utilizzata nelle precedenti ricerche sulla classe politica municipale, in modo da permettere la comparazione di dati vecchi e nuovi. Circa i criteri di costruzione delle categorie della stratificazione, si rinvia a G. Bettin, A. Magnier (1989) in particolare alle note 1, 5, 20 (pp. 95, 98, 114). La nomenclatura utilizzata nelle nostre ricerche consente un confronto parziale con $\mathrm{i}$ dati del Ministero, ma ci permette di approfondire una riflessione più dettagliata su alcune categorie cruciali (quella dei liberi professionisti ad esempio). L'uso di una doppia domanda semi-aperta, sulla professione attuale e sulla professione al momento dell'ingresso in politica, nel questionario rivolto ai nostri due campioni di sindaci è stato fruttuoso nel contesto della scoperta.
} 
per i più è riservato ad un'età più matura; al contempo, il candidato libero professionista non è attratto da una posizione di pubblica responsabilità nel periodo in cui, di solito, il prestigio professionale privato tende ad essere massimo - cioè dopo $\mathrm{i}$ cinquant'anni.

Tutti sappiamo che la carriera di un politico, e non solo questa, viene condizionata nelle sue manifestazioni dalle risorse di tipo ascrittivo e da quelle di tipo acquisitivo che lo definiscono socialmente. Tra le risorse del secondo tipo spicca l'istruzione, per l'influenza che ha sulla mobilità e sugli aspetti dinamici, in generale, della carriera. I dati sui nuovi sindaci, nonostante il terremoto elettorale e l'affermarsi dei movimenti localistici, confermano il trend premiante per il primo cittadino ufficialmente colto. Più della metà (il $53,5 \%$ ) dei nuovi sindaci risulta aver conquistato una laurea prima di accedere allo scranno; il $9 \%$ ha un diploma liceale; il $28,7 \%$ dichiara di avere un altro tipo di diploma di scuola media superiore; il 7,4\% ha concluso gli studi con le medie inferiori e, infine, solo l' $1,3 \%$ ha la licenza elementare. I due terzi delle donne elette sindaco nel 1993 sono laureate. Si può allora dire che, con riferimento allo sparuto drappello dei sindaci donna $(6,3 \%$ dei nuovi sindaci), l'istruzione opera come fattore di incremento della carriera politica, contrastando con successo la pressione discriminatoria verso il personale politico femminile che altro non è se non uno tra i molti segnali di vischiosità e di antimodernità della nostra cultura politica.

L'analisi diacronica dei dati sulla posizione professionale dei membri della classe politica municipale risulta difficile per il cambiamento di nomenclatura adottata, in particolare riguardo ad alcune categorie di dipendenti pubblici. Confrontando i dati del 1992 sulla posizione professionale dei sindaci in carica con quelli di cinque anni prima, è tuttavia possibile osservare che le due categorie dei «servizi» e del «pubblico impiego» passavano complessivamente dal 61,3\% del 1987 al 77,9\% del 1992. E ancora che i sindaci occupati nella categoria dei «servizi» erano nel frattempo cresciuti numericamente alquanto più degli occupati nel «pubblico impiego» ${ }^{10}$. Dalle nostre rilevazioni del 1993,

${ }^{10} \mathrm{La}$ differenza tra il settore terziario e quello dei dipendenti pubblici non risulta molto agevole alla luce della classificazione ISTAT. Le categorie professionali degli insegnanti, ad esempio, sono inserite tout-court tra i servizi (e non tra i dipendenti pubblici). Così pure è per i medici, inseriti nel settore dei servizi, senza distinzione tra profes- 
si constata tra i nuovi sindaci una leggera contrazione del gruppo «servizi e pubblico impiego» $(72 \%)$, da attribuirsi quasi in toto al calo della quota dei lavoratori dipendenti del settore dei servizi. Gli alfieri del processo di trasformazione del personale politico sono, nel 1993, soprattutto gli esponenti di alcune professioni liberali. Tra questi, i medici (l'11,7\% dei sindaci italiani) sembrano essere i maggiori depositari della crescita di fiducia concessa ai candidati attivi nel settore del lavoro indipendente. Anche gli insegnanti paiono avere maggior credito, seguiti da geometri, ingegneri e architetti. Per gli avvocati e per i commercialisti non si registra, invece, una tendenza analoga. Le professioni ad alta specializzazione giuridica, se svolte nel settore privato, non riscuotono il successo che viene invece tributato nell'arena politica ai detentori di ruoli percepiti come ruoli caratterizzati da universalismo, da uno status di imparziale autorità (i professori) e da una vocazione di servizio (i medici) ${ }^{11}$. Sembrava, parimenti, da menzionare nel 1992 il rinnovato - anche se non consolidato nel tempo - potere attrattivo dei candidati sindaci operai: i sindaci «in tuta blu» raddoppiavano rispetto al 1987 (dal 3,3\% al 6,6\%). Il fenomeno riguardava quasi completamente $\mathrm{i}$ centri minori ed era un indizio apparente di una ripresa di vitalità e di interesse per la politica da parte di alcuni strati sociali che ne erano stati a lungo estromessi. La tendenza però si arresta nel 1993, assorbita in un processo più ampio che, per comodità, si può etichettare «imborghesimento» dei primi cittadini.

L'esame ravvicinato dei dati sui nuovi sindaci ci dice che il quasi $40 \%$ di sindaci borghesi è costituito da un $1,3 \%$ di docenti universitari, un $4,1 \%$ di imprenditori, un $9,4 \%$ di dirigenti di enti pubblici e di aziende private, e da ben il $23,9 \%$ di liberi professionisti. Il $50,9 \%$ dei nuovi sindaci considera che la sua «professione abbia influito positivamente sulla sua elezione» («molto» o «in parte»); la proporzione è assai maggiore tra i liberi professionisti $(68,6 \%)$, minore, ma ancora superiore alla media, per gli imprenditori $(60 \%)$ e i dirigenti $(58 \%)$. In que-

sionisti che operano in strutture pubbliche e quelli occupati in strutture private. La voce 'dipendenti pubblici' è forse più corposa di quanto non risulti seguendo la classificazione in oggetto.

${ }^{11}$ Tra i sindaci eletti nelle due tornate elettorali immediatamente successive alla riforma, comunque, avvocati e notai sono più numerosi di prima $(5 \%)$. 
ste valutazioni, lo status complessivo della professione sembra combinarsi ma anche predominare sulle possibilità di relazioni che essa offre: una tipica professione da contact man, quella dell'insegnante, supera a malapena la media $(54,9 \%)$. Contrariamente all'immagine tradizionale del mondo rurale, come retaggio di un personale politico proveniente da un notabilato di cui il prestigio professionale era una componente importante, ma conformemente a molte osservazioni precedenti e a conferma della scomparsa di questo notabilato tradizionale, l'importanza attribuita alla professione è ridotta nei microcomuni dove la conoscenza interindividuale è più intensa ${ }^{12}$.

I sindaci «borghesi» di oggi, in genere, lo erano già quando hanno iniziato a fare politica. La mobilità verticale deducibile dal confronto tra la posizione professionale dei sindaci «prima di iniziare a far politica» e «oggi» è molto più ridotta di quanto si potesse prevedere. La stabilità è la regola e concerne il $93,8 \%$ dei casi. Una mobilità ascendente si rileva soltanto nel $5,5 \%$ dei casi. In quattro casi, vi sarebbe pure mobilità discendente. Al livello del sindaco, far politica ha a volte consentito il passaggio da un settore di attività meno protetto ad un settore più «garantito» e controllato dai partiti, ma non sembra produrre - almeno fino a questo punto della carriera - una vera $e$ propria promozione professionale.

Rimaneva e rimane comunque confermata, anche se è minacciata fortemente dall'avanzata dei «borghesi», la preponderanza dei ceti medi dipendenti che, con il $40,5 \%$ di rappresentanti tra i nuovi sindaci, costituiscono pur sempre il serbatoio di reclutamento più produttivo di politici municipali. Se consideriamo i settori di attività, osserviamo che la parte del leone continua ad essere giocata dai dipendenti pubblici, che esprimono attorno ai due quinti dell'intera popolazione dei sindaci in carica e occupati ${ }^{13}$. In sintesi, l'area sociale di reclutamento dei sindaci sembra rimanere nel suo complesso piuttosto stabile anche se l'interesse della borghesia per il governo dei municipi

\footnotetext{
${ }^{12}$ Essa aumenta da una quota del $41,5 \%$ di giudizi positivi sulla sua influenza nei microcomuni, ad una quota del $66,4 \%$ nelle città medie, per scendere al $63,6 \%$ nei comuni sopra i 30.000 abitanti.

${ }^{13}$ Le differenze geografiche rimangono forti al riguardo: questa stessa quota di sindaci occupati nel settore pubblico o parapubblico, «lottizzato», varia da circa la metà dei sindaci nel Centro Italia ad un quarto nelle regioni settentrionali; le regioni meridionali si situano in una posizione intermedia, i due quinti circa.
} 
TAв. 2. Appartenenza partitica e livello sociale dei nuovi sindaci (\% di riga)

\begin{tabular}{|c|c|c|c|c|c|c|}
\hline \multirow[b]{2}{*}{ PARTITO } & \multicolumn{5}{|c|}{ LIVELLO SOCIALE } & \multirow[b]{2}{*}{ Totale } \\
\hline & Borghesia & $\begin{array}{c}\text { Piccola } \\
\text { borghesia } \\
\text { dipendente }\end{array}$ & $\begin{array}{l}\text { Piccola } \\
\text { borghesia } \\
\text { indipend. }\end{array}$ & Operai & N.d. & \\
\hline Ppi & 36,4 & 46,6 & 5,7 & 1,7 & 9,7 & 100,0 \\
\hline $\begin{array}{l}\text { Pds } \\
\text { Rifondaz. }\end{array}$ & 26,0 & 50,9 & 4,1 & 3,0 & 16,0 & 100,0 \\
\hline $\begin{array}{l}\text { Psi } \\
\text { Psdi }\end{array}$ & 42,2 & 39,1 & 1,6 & 1,6 & 15,6 & 100,0 \\
\hline Leghe & 44,6 & 28,6 & 5,4 & 0,0 & 21,4 & 100,0 \\
\hline $\begin{array}{l}\text { Altri } \\
\text { partiti }\end{array}$ & 38,6 & 43,2 & 0,0 & 0,0 & 18,2 & 100,0 \\
\hline Emancipati & 47,8 & 35,4 & 6,2 & 0,9 & 9,7 & 100,0 \\
\hline Orfani & 28,6 & 40,5 & $\begin{array}{l}2,4 \\
?\end{array}$ & 0,0 & 28,6 & 100,0 \\
\hline Outsiders & 46,5 & 31,9 & 5,4 & 0,0 & 16,2 & 100,0 \\
\hline
\end{tabular}

I sindaci emancipati hanno avuto in passato una tessera, ma già nel 1991 non erano più iscritti ad un partito; i sindaci orfani hanno cessato di essere iscritti nel 1991, 1992 o 1993; i sindaci outsiders dichiarano di non essere mai stati iscritti ad un partito.

Fonte: Nostro campione: 865 sindaci eletti nel 1993.

costituisce una novità veramente interessante e forse non è soltanto il frutto dell'affermarsi sulla scena di movimenti di matrice localista, come dimostra la tabella 2 .

Sindaci di partito o sindaci di paese?

Malgrado le trasformazioni che hanno investito, a ritmi serrati, il sistema partitico italiano in questo primo scorcio degli anni Novanta, un'analisi del rapporto che lega i sindaci in carica alla vigilia della riforma elettorale con i partiti che li hanno sostenuti nell'elezione conserva un interesse non secondario in vista di successive e auspicabili verifiche sugli effetti innovativi 
dovuti alla legge 81 . La distribuzione dei posti di sindaco tra $\mathrm{i}$ partiti alla fine del 1992, prima delle due tornate elettorali che hanno prodotto i nuovi sindaci, offre un'immagine della politica italiana forse più simile a quella di vent'anni prima che a quella di venti mesi dopo. All'istante in cui tale fotografia è stata scattata, la DC si assicurava il 54,2\% dei sindaci; di gran lunga distanziati seguivano tutti gli altri partiti. I sindaci democristiani costituivano la maggioranza in sedici regioni su venti, escluse le «regioni rosse», dove le roccaforti comuniste non sembravano dare seri segni di crisi, e la Val d'Aosta ${ }^{14}$. In Veneto il $77,7 \%$ dei sindaci si era insediato sotto l'insegna del «bianco fiore». Ancora: non va dimenticato che nel Nord del Paese, il PSI si presentava come il partito con il maggior numero di sindaci dopo la DC.

Solo un anno dopo, lo scenario è sconvolto. Anche lo studioso si trova in difficoltà a mettere in ordine $i$ dati sulle ultime elezioni ove si affidi unicamente all'anagrafe ministeriale. Basti ricordare che l'eterogeneità delle coalizioni elettorali nei municipi coinvolti dalla legge 81 e la moltiplicazione delle sigle non consentono facilmente di codificare le coalizioni elettorali né di situare con precisione la collocazione di molti sindaci ${ }^{15}$. Il nostro campione ci permette, invece, di collocare il sindaco eletto nell'ambito di una formazione politica specifica, sulla base dell'affiliazione dichiarata da ciascun intervistato. Ne emerge chiarissimo il distacco - vero o di facciata - dal sistema dei partiti che il nuovo sindaco intende comunicare. Alla domanda «Lei è iscritto o è stato iscritto a qualche partito politico?», il $40 \%$ dei nuovi sindaci non risponde o afferma di non essere iscritto ad un partito politico ${ }^{16}$. Tra costoro si identifica un gruppo consistente di eletti (il 18,2\% del campione) accomunati da un'esperienza biografica significativa: non sono iscritti ad alcun partito, $\mathrm{ma}$ lo sono stati in passato. É possibile distinguere ulteriormen-

${ }^{14}$ In questa regione, le liste civiche o collegate alla Union Valdôtaine esprimevano il $60,8 \%$ dei sindaci, cui va aggiunto un $24 \%$ di eletti indipendenti in varie liste. (1994).

${ }^{15}$ Un utile ed approfondito tentativo di lettura dei dati si trova in A. Di Virgilio

${ }^{16} \mathrm{La}$ quota di sindaci apartitici appare superiore a quella risultante dai dati offerti dall'anagrafe ministeriale sui nuovi sindaci, in cui $i$ «non iscritti» sono suddivisi in varie categorie: gli «indipendenti» tout court, gli indipendenti eletti nella lista dei grandi partiti, i dissidenti degli stessi partiti, gli eletti nelle liste civiche. La somma degli appartenenti a queste categorie raggiunge il $30,7 \%$. 
te, all'interno di questo gruppo, tra gli emancipati (coloro che hanno lasciato il partito prima del 1991), che rappresentano ben il $13,3 \%$ dei nuovi sindaci, e gli orfani (coloro che hanno lasciato il partito tra il 1991 e il 1993), che rappresentano il $4,9 \%$ degli intervistati. Se si esclude questa schiera folta di apolitici e politici «pentiti», i Popolari mantengono il primato del potere municipale con il $20,7 \%$ dei nuovi sindaci (quota che corrisponde alla metà della quota di potere detenuto dalla vecchia DC). Il PDS, insieme a Rifondazione comunista, raggiunge il 19,9\%; il PSI, insieme al PSDI, raggruppa solo il 7,5\%; le Leghe il 6,6\%; gli altri partiti il 5,2\%.

Questi pochi dati fondamentali sono sufficienti per intravvedere l'apertura di una nuova fase nel sistema politico periferico del nostro Paese all'insegna di una netta autonomia nella carriera del politico: sarebbero promossi quei candidati che sanno utilizzare al meglio il sostegno instabile dei partiti, di nuova e vecchia formazione, che li hanno selezionati. Tuttavia, il sociologo della politica non può non rimarcare che le procedure di selezione del personale politico rimangono sostanzialmente inalterate. I partiti, in una forma più o meno trasparente, continuano ad essere la matrice principale di queste procedure, mentre la società civile resta separata e non riesce ancora a farsi rappresentare in una maniera adeguata. Ne è indizio il $38,6 \%$ dei nuovi sindaci che dichiara la propria assoluta estraneità al mondo delle associazioni professionali, sindacali, di volontariato, culturali, parrocchiali o comunque espressioni della società civile.

Tutti gli studiosi di comportamento elettorale sanno che prima della riforma della legge 81 il posizionamento dei candidati nella lista costituiva ovunque un indicatore-chiave per comprendere come il partito conducesse il suo gioco per ottenere un proprio rappresentante sulla poltrona di sindaco. Due le diverse strategie di fondo che sembravano essere possibili: mettere in risalto, con largo anticipo sui tempi e sui risultati, il sindaco da proporre in una fase successiva di contrattazione, oppure lasciare aperta la competizione interna e l'eventuale inserimento di outsiders nascosti nelle pieghe dell'ordine alfabetico della lista. Ora, dai dati del servizio elettorale del Ministero dell'Interno riguardanti i sindaci in carica all'inizio del 1993 , ossia alla vigilia della riforma, appare che il 40,6\% dei futuri sindaci occupava il primo posto di lista e il $33,2 \%$ il secondo. Il rimanente $16 \%$ era diluito in concentrazioni via via più blande 
nelle posizioni successive ${ }^{17}$. Si deve, al contempo, evidenziare che in quasi tutti i partiti esaminati lo stacco tra le prime due posizioni e le rimanenti era fino al 1993 così netto che sembra legittimo interpretare la collocazione in testa di lista come una sorta di candidatura a sindaco, già prima, si noti, della riforma maggioritaria. Su questo punto, dunque, la legge per l'elezione diretta del sindaco è andata a incrinare delle norme comportamentali consolidate all'interno delle formazioni politiche, per la conquista del potere locale. Sarebbe interessante effettuare un'analisi sull' «effetto-traino» dei primi candidati sull'intera lista (in base alla percentuale di voti di preferenza del sindaco sul voto complessivo della sua lista), ma i dati ministeriali non consentono tanto. È possibile, tuttavia, distinguere alcune significative differenze regionali relative all'attitudine dei partiti a proporre candidature forti, molto personalizzate, di futuri sindaci.

Comunque fosse, prima della riforma, la derivazione partitica della candidatura era massima: la sola differenza stava nel momento in cui tale candidatura veniva alla superficie - prima o dopo la competizione elettorale. A conferma di tale tesi, gioca anche la scarsa importanza che rivestivano i sindaci che si dichiaravano ufficialmente candidati «indipendenti» tra i sindaci in carica all'inizio del 1993: essi costituivano solo il $12 \%$ del totale. L'importanza degli indipendenti assumeva caratteri minimi nei tre partiti storici della politica italiana, con la punta più bassa nell'ex PCI. Solo nel PLI il $25 \%$ dei sindaci non era iscritto. Fino al 1993, la figura dell'indipendente meritava una particolare attenzione, per comprendere se essa era una candidatura in evoluzione, tentativo individuale di accesso alla carica che scavalcava la costrizione dei ruoli all'interno dei partiti, o se rappresentava un fenomeno marginale a cui i partiti hanno fatto a più riprese ricorso per «imbiancare la facciata». Geograficamente, il fenomeno interessava particolarmente le aree più pro-

\footnotetext{
${ }^{17}$ Anche se si volesse ipotizzare che il capolista rappresenti il fiore all'occhiello delle candidature presentate, i dati rilevati non autorizzano questa interpretazione. Sembra, anzi, dai nostri dati sui sindaci eletti, che il candidato che funge da capolista non spicchi per una più alta istruzione o per particolari caratteristiche individuali, sia biografiche (età, genere) sia socioeconomiche (settore occupazionale, professione). La scelta del capolista, dunque, sembrava prescindere totalmente dal prestigio sociale dell'individuo prescelto, confermando l'impressione che nella formazione delle liste municipali il merito politico facesse premio sul merito sociale.
} 
duttive e ricche del Paese, visto che, nel 1992, su 958 sindaci eletti come indipendenti, 618 erano del Nord Ovest; staccati, di molto, seguivano il Sud, il Centro e il Nord Est, dove le personalità non inquadrate costituivano solo il 7,9\% dei sindaci. La distribuzione regionale degli eletti indipendenti era, comunque, influenzata dalla distribuzione sul territorio dei comuni minori. Il $98 \%$ dei sindaci indipendenti era al governo di centri con popolazione inferiore ai diecimila abitanti. Forse, quindi, la piccola comunità ha mostrato per prima una marcata insofferenza ai legami partitici. Per quel che riguarda la collocazione sociale, non vi erano particolari divergenze dalla distribuzione professionale dell'insieme dei sindaci. Evidentemente, il desiderio di essere protagonisti fuori dai partiti era diffuso in tutti gli strati sociali. Oltre a quanto si è detto sul rifiuto della tessera da parte degli aspiranti sindaci presentatisi nelle liste dei partiti tradizionali, già alla vigilia della riforma del 1993 andava tenuto in conto l'incremento dei primi cittadini eletti in formazioni politiche nuove. Esse hanno espresso il 10\% dei sindaci in carica all'inizio del 1993 ed hanno rappresentato, insieme al fenomeno dei sindaci non iscritti ai partiti, una voce considerevole della richiesta di cambiamento delle regole dal basso.

Eppure ancora nel 1993 il rapporto con la società civile sembra mediato da quelle speciali macchine promotrici di consenso che sono i partiti. Alla domanda: «Nella campagna elettorale ha avuto l'appoggio di...?», un nuovo sindaco non iscritto su cinque dichiara di avere avuto un appoggio «forte» o «sufficiente» dal partito, proporzione bassa rispetto alla media del campione $(44,9 \%)$, ma sorprendente nella misura in cui è espressa da un personale che avrebbe preso le distanze dal sistema dei partiti. Tutte le categorie di non iscritti si sono appoggiate ad un «comitato elettorale creato appositamente», ben più dei colleghi «allineati»; tutte hanno fatto grande ricorso all'appoggio degli «amici» (in particolare gli emancipati: il 64,6\% di loro indica «gli amici» come una «forte» fonte di sostegno, il $27,4 \%$ come un sostegno «sufficiente»). Nel complesso, ne esce confermata un'immagine molto sbiadita della società civile come sostenitrice di candidature vincenti, mediante le associazioni professionali e i sindacati o il «mondo dell'economia». 
Le due facce della carriera del sindaco: incarichi di partito e cariche elettive

Prima della legge 81 , la classe politica municipale risultava articolata in tre livelli intercomunicanti (consiglieri, assessori e sindaci), tendenzialmente omogenei sotto il profilo della carriera politica. Le ricerche precedenti del Centro Interuniversitario di Sociologia Politica sui consiglieri e sugli assessori delle città suffragavano la tesi del «ceto politico semiautonomo» (Segatori 1992). I dati sui sindaci in carica nel 1992 offrivano una conferma di questa tesi. Anche nei comuni minori, in Italia era pressoché impossibile diventare sindaci ex abrupto, senza avere ricoperto una qualche carica politica in precedenza. A riuscire in questa impresa eccezionale era solo il $2,3 \%$ dei primi cittadini di allora. Ma v'è di più: se non impossibile, era anche difficile arrivare al vertice del comune senza essere stati eletti in una legislatura precedente (vi riusciva solo $1^{\prime} 8,5 \%$ dei sindaci) o senza avere avuto prima un incarico di partito (il 16\% dei sindaci). Molto ridotto era il numero dei non iscritti a un partito $(4,3 \%)$; esiguo quello degli iscritti da meno di dieci anni $(11,1 \%)$. In media, i primi cittadini avevano accumulato trentuno anni di attività politica, di cui quindici negli incarichi di partito, tredici in posti elettivi, e tre in organi di nomina politica (aziende municipalizzate, unità sanitarie locali, e simili) ${ }^{18}$. In generale, dunque, valeva per i sindaci 1992 ciò che si era riscontrato per gli assessori nel 1990: a) il ruolo di sindaco era una tappa di una carriera, e non una esperienza episodica; $b$ ) questa carriera aveva il suo baricentro nel partito. Insomma, l'esperienza politica, e partitica in particolare, era la componente determinante per aspirare alla poltrona di sindaco. Nonostante che solamente il $7,4 \%$ dei primi cittadini che aveva risposto al questionario nel 1992 si autodefinisse «politico di professione», la durata temporale pregressa, la profondità dell'impegno profuso nella carica di sindaco (che occupava una media di quarantanove ore settimanali) e l'ampiezza complessiva del coinvolgimento - attuale e passato - in incarichi di governo e di partito di questa frazione della classe politica testimoniavano efficacemente di come il professionismo politico fosse per la maggior parte dei sindaci

\footnotetext{
${ }^{18}$ Il dato deriva dalla somma degli anni passati nei diversi incarichi, in sequenza o parallelamente.
} 
italiani un requisito di eleggibilità alla carica, e per quasi tutti un approdo difficilmente evitabile.

Con l'elezione diretta dei sindaci, i dati riguardanti la durata della carriera pregressa si modificano soltanto in parte. La proporzione di sindaci che ama dichiararsi «politico di professione» si è ulteriormente ridotta $(1,7 \%)$. Il numero dei non iscritti, lo abbiamo visto, è notevolmente aumentato. La proporzione di sindaci che si candidano al buio, da neofiti, senza nessuna esperienza politica (nel partito, elettiva, in organi di nomina partitica) passa dal $2,3 \%$ al $24,2 \%$. L'esperienza in consiglio comunale è spesso richiesta, ma non si presenta più come una tappa obbligata (il 53\% ne è esente). La media dell'esperienza politica in senso lato (in incarichi partitici, elettivi o amministrativi) rimane significativa, poiché supera i 16 anni, ma si è dimezzata rispetto al 1992. Quest'esperienza si è ridotta nelle sue varie componenti: la durata media degli incarichi amministrativi ricoperti non raggiunge i due anni, quella degli incarichi di partito i sette anni, quella delle responsabilità di rappresentanza elettiva si attesta sugli otto anni.

Il percorso di carriera risultante dall'indagine del 1992 non era affatto lineare. Erano rare le carriere su un solo binario carriere, cioè, sviluppate unicamente all'interno del partito $(4,9 \%)$ o delle assemblee elettive $(9,5 \%)$. Si può leggere questo dato solo in una chiave: poter contare su un background politico variato metteva in grado di controllare meglio, in qualche misura, sia i filtri selettori del partito che quelli dell'istituzioneComune. Non va trascurato il fatto che poter chiamare in causa l'esperienza amministrativa maturata al vertice di enti pubblici (le unità sanitarie locali, per esempio) significa per il candidato al ruolo di sindaco disporre di un bagaglio considerevole di competenza e, non di rado, di voti personali (1.213 preferenze in media per i sindaci interrogati nel 1992). In generale, sembra che l'articolazione della carriera pregressa fosse un requisito tanto più importante quanto più grande era l'importanza del comune da amministrare. Il curriculum misto era una prerogativa che assumeva rilevanza maggiore all'aumentare delle dimensioni del comune. Viceversa, nei centri con meno di cinquemila abitanti, sia pure in una misura molto relativa, era più probabile che il sindaco non avesse alcuna esperienza politica precedente $(3,8 \%$ nel 1992 contro una media generale del $2,3 \%)$, o soltanto un'esperienza come consigliere o assessore comunale (15\% nel 1992 contro una media generale del 9,5\%), oppure 
soltanto un'esperienza in un organo di partito ( $11 \%$ contro una media generale dell' $8,5 \%$ ). Queste cifre erano tuttavia basse e denunciavano già prima della riforma una tendenza importante sulla quale torneremo. Notiamo per ora che la carriera composita era più tipica degli ex operai, dei tecnici, dei non attivi (pensionati e studenti). I sindaci che invece avevano alle spalle uno status professionale più alto (dirigenti pubblici e privati, liberi professionisti, insegnanti, commercianti) si potevano permettere di accedere alla carica con un patrimonio più ridotto di esperienze politiche precedenti e, soprattutto, con una carriera dal raggio meno vasto e meno eterogenea (o soltanto nel partito, o soltanto in incarichi elettivi). Allo stesso modo, i laureati risultavano leggermente avvantaggiati rispetto ai meno scolarizzati per diventare sindaci senza una carriera politica dietro di sé, o comunque senza aver ricoperto alcun incarico di partito. E ancora: chi diceva di essere entrato in politica sulla scia di un familiare $\mathrm{o}$ di un altro personaggio che già aveva ricoperto qualche carica d'autorità tendeva ad arrivare al vertice del municipio con un curriculum più limitato e meno differenziato in termini di incarichi politici, perché utilizzava principalmente le risorse tipiche di un notabilato che si tramandava da una generazione all'altra. Tutti questi elementi convergevano nel giustificare una prima conclusione: l'articolazione della carriera politica pregressa valeva, almeno fino al 1993, come una risorsa compensativa di altre risorse personali spendibili nella competizione per l'accesso alla carica di sindaco. Un iter variato era ciò che serviva agli aspiranti sindaci più deboli in partenza per acquisire quelle capacità e quelle conoscenze che ad altri erano date per nascita, studi, amicizie precedenti, mestiere.

Accanto ai condizionamenti istituzionali, che fanno della carriera un equivalente funzionale dello status sociale di partenza, entravano poi in gioco elementi soggettivi - come le motivazioni all'agire politico - desumibili da vari indicatori, anche se di misurazione empirica tutt'altro che agevole. L'età di ingresso nella politica attiva, per esempio, può essere considerata un indicatore della volontà di fare della politica una professione. Non è casuale che chi entrava molto giovane in politica tendesse ad occupare fin dall'inizio contemporaneamente diverse cariche (nella sezione, nel consiglio di quartiere o comunale, nel movimento giovanile del partito, in un consiglio d'amministrazione di nomina politica). Ebbene: quanto più precoce era il futuro sindaco nel ricoprire un incarico politico, tanto più artico- 
lato risultava il suo cursus honorum complessivo. Al contrario, chi entrava tardi in politica (ossia, dopo i trent'anni) compiva un'esplorazione meno capillare delle posizioni di potere fuori e dentro il partito, limitandosi per lo più ad inserirsi in una filière specifica (il consiglio comunale o gli organi locali di partito). Se ne può concludere che carriere più lunghe ed articolate connotavano i sindaci professionisti della politica, per i quali la poltrona di primo cittadino sembrava essere più una tappa in un percorso di ascesa personale che una vocazione specifica. Riprova ne era che tutti i sindaci studiati che si autodefinivano «politici di professione» provenivano da una carriera politica mista - partitica e istituzionale. Per il dopo 1993, valgono le stesse osservazioni?

I dati raccolti sui nuovi sindaci autorizzano, come abbiamo visto, l'idea di un rinnovamento del personale politico eletto con l'ingresso di bomines novi appartenenti a formazioni nuove. Fra i sindaci non neofiti, poi, quasi un terzo non ha mai vissuto la vita quotidiana da dirigente responsabile di partito. Tuttavia, l'ipotesi di una rivalutazione della carriera elettiva non è per ora realmente avvalorata; l'esperienza elettiva media supera di poco l'esperienza di partito (8 anni contro 7). La gavetta interna al partito è stata la scuola ancora per molti sindaci di tutte le formazioni, al di là delle tradizionali differenze.

\section{Mobilità e temporalità nella carriera politica}

Le variabili che incidono sulle modalità della carriera politica sono, come abbiamo visto, molteplici; al fine di individuare quelle determinanti per la definizione di una tipologia che aggreghi in una forma esaustiva i dati pertinenti il nostro campione, la variabile «durata» assume però una valenza cruciale e merita un esame più sistematico. Le tipologie possibili sono tante, se non altro perché le tappe costitutive della carriera politica si sviluppano secondo schemi molto differenziati che associano passaggi obbligati a posizioni facoltative, più diretta espressione della libera scelta dell'attore, delle sue risorse politiche personali e dei suoi disegni di carriera. D'altro canto, al livello periferico assai più che al livello centrale, la mappa delle posizioni appetibili per un politico di professione è in profonda ridefinizione. Al tempo stesso, ciascuna posizione ha un grado di influenza che varia anche in relazione alla possibilità di resa 
oggettiva della carica e a seconda dell'interpretazione che ne dà l'attore che la ricopre. Usualmente chi studia il professionismo politico e si preoccupa di misurarne l'efficacia valorizza al massimo la distinzione tra cariche elettive e incarichi di partito al fine di lumeggiare l'ipotesi partitocratica che, tuttavia, sembra oggi assai meno pregnante sul piano euristico di altre. Tale ipotesi ci ha permesso finora di ponderare l'importanza relativa di certi tipi di carriera e di certe posizioni propedeutiche. La durata, come abbiamo visto, differenzia fortemente i percorsi all'interno di questi schemi. Chi si dedica all'analisi specifica della carriera come processo formativo del personale politico professionalizzato deve organizzare i suoi dati empirici secondo parametri unificanti. La temporalità, allora, assume in tutta la sua evidenza il valore di variabile-chiave utile ad una lettura discriminante dei percorsi altrimenti difficili da classificare. Il politico di professione vive come qualsiasi cittadino in un tempo istituzionale connesso alla durata dell'incarico normativamente definita. Il tempo istituzionale è, ovviamente, vissuto in un perenne confronto con il tempo personale dell'attore in carriera. Il tempo personale è la risultante di una pluralità di componenti tutte riconducibili alle interpretazioni soggettive dei ruoli nonché all'intreccio tra status personale, congiuntura politica generale, capacità e risorse spendibili dall'attore in vista dei suoi obiettivi e dei suoi interessi. Utilizzando la dimensione-tempo come parametro fondamentale per valutare la carriera politica si riesce a comprendere meglio quanto contino alcune risorse investite nella carriera e forse anche si intravvede il disegno di carriera che motiva l'attore e che ne orienta il movimento.

Il dato di base che può orientare la nostra analisi fondata sulla temporalità è fornito dal $41 \%$ di sindaci in carica nel 1992 che aveva alle spalle oltre un quarto di secolo di carriera politica (ossia, in qualche ruolo elettivo o di partito). Accanto a questi politici professionisti ultracollaudati si poneva un secondo gruppo di dimensione più che rispettabile (il $21 \%$ ) che aveva iniziato la carriera politica tra il 1967 e il 1974. Un altro $24 \%$ di sindaci aveva cominciato la sua esperienza fra il 1975 ed il 1986; assai più contenuto era il gruppo dei sindaci la cui anzianità di carriera non superava i cinque anni (13\%). Solo il 3\% aveva esordito in politica proprio con la carica apicale di sindaco. Tra i sindaci eletti nel 1993, la proporzione di veri principianti, che dichiarano addirittura di essere entrati in politica candidandosi direttamente alla guida del comune diventa significativa, seppure mino- 
ТАв. 3. I canali e la durata della carriera politica dei sindaci (campioni sindaci 1992 e 1993)

\begin{tabular}{lrr}
\hline HANNO RICOPERTO CARICHE: & 1992 & 1993 \\
\hline Elettive & 90,8 & 64,1 \\
& & \\
$1-2$ anni & 4,9 & 2,8 \\
3-5 anni & 14,0 & 17,0 \\
oltre 5 anni & 71,9 & 44,3 \\
& & \\
Partitiche & 83,7 & 49,3 \\
& & \\
$1-2$ anni & 8,5 & 6,4 \\
3-5 anni & 16,4 & 9,0 \\
oltre 5 anni & 58,8 & 33,9 \\
& & \\
Amministrative & 41,2 & 21,1 \\
$1-2$ anni & 8,8 & 5,3 \\
3-5 anni & 17,0 & 8,6 \\
oltre 5 anni & 15,4 & 7,2 \\
\hline
\end{tabular}

La 'durata' corrisponde alla somma degli anni dedicati ai singoli incarichi (anche se svolti simultaneamente).

ritaria: un quarto circa. La concentrazione nelle formazioni politiche nuove lascerebbe supporre tuttavia che il fenomeno corrisponde all'emergenza di movimenti in rottura, anche per quel che concerne le forme del reclutamento, con il sistema politico preesistente, più che corrispondere ad una conseguenza vera $\mathrm{e}$ propria dovuta alle innovazioni istituzionali.

Oggi come ieri, tuttavia, la pluralità delle esperienze e la frequente mobilità da una carica all'altra caratterizzano in forma forte la formazione del sindaco italiano medio. Ove si voglia approfondire in maniera più analitica il tema, grazie ai dati rilevati, si osserverà che all'anzianità crescente nella carriera corrisponde sempre un numero crescente di cariche ricoperte. In altri termini, il sistema politico periferico configura la carriera come una sequenza di molte posizioni ricoperte successivamente ad intervalli di tempo abbastanza ristretti. Non è certo il caso di interpretare il passaggio da un gradino di carriera all'altro come una perversa vocazione alla perdita delle risorse accumulate nella posizione di influenza precedente. Il politico in carrie- 
ТАв. 4. Le tappe della carriera politica dei sindaci (campioni sindaci 1992 e 1993)

\begin{tabular}{lrr}
\hline & 1992 & 1993 \\
\hline IN INCARICHI PUBBLICI & & \\
ELETTIVI: & & \\
& 56,5 & 49,5 \\
Consigliere comunale & 51,3 & 28,7 \\
Assessore & 0,0 & 5,9 \\
Consigliere provinciale & 1,0 & 0,7 \\
Consigliere regionale & 2,6 & 1,6 \\
Parlamentare & & \\
& & \\
IN PARTITI POLITICI: & & \\
& 41,9 & 27,6 \\
Segretario di sezione & 29,9 & 16,9 \\
Membro direttivo provinciale & 9,0 & 4,3 \\
Membro direttivo regionale & 0,6 & 3,4 \\
Membro direttivo nazionale & & \\
& & \\
IN INCARICHI PUBBLICI & & \\
NON ELETTIVI: & & \\
Comitato gestione USL & 17,3 & 7,5 \\
Cons. azienda servizi pubblici locali & 6,9 & 2,3 \\
Consiglio IPAB & &
\end{tabular}

ra comprende l'importanza di una crescita rapida e continua, un perpetuum mobile tale da consentirgli di costruire selettivamente una sua rete relazionale. Come il pattinatore sul ghiaccio appena formato, il politico di professione sa che le sue chances di sopravvivenza aumentano se non si sofferma troppo su una posizione e vive la sua carriera come un incessante passaggio da una mèta all'altra: l'impegno per la mobilità, naturalmente, si orienta verso un itinerario ascendente, percorrendo il quale ci si imbatte in un numero crescente di attori in competizione tra di loro per uno spazio politico che, invece, si restringe via via che si procede verso le cariche apicali.

\section{Verso un declino della politica come carriera?}

Per concludere, riassumiamo alcuni tratti fondamentali delle carriere dei sindaci così come abbiamo potuto ricostruirle grazie ad una rilevazione in due fasi, nel 1992 e nel 1993, prima e dopo l'applicazione della legge 81 . Innanzitutto la carriera del 
sindaco prima della riforma del 1993 non è fondamentalmente diversa da quella dell'assessore. Il sindaco appare come un primus inter pares, un politico locale più capace di altri suoi compagni di strada. Il fenomeno degli bomines novi, accuratamente misurato, si riduceva al $2,3 \%$ dei sindaci nel 1992 . I neofiti veri e propri costituiscono, invece, quasi un quarto dei sindaci eletti nel 1993. Nella più parte dei casi, però, ancor oggi quando la carriera pregressa del primo cittadino ha visto un passaggio nelle strutture di partito, si è trattato di una vera e propria gavetta, iniziata addirittura dall'incarico di segretario di sezione (così è per oltre il $25 \%$ dei nuovi sindaci).

L'esperienza di una carriera politica in genere assume configurazioni specifiche in relazione ai contesti, istituzionali e non, che accompagnano il suo sviluppo. I partiti promuovono ancora i loro sindaci seguendo degli schemi di reclutamento alle diverse cariche che non sono omogenei e in molti casi senz'altro mascherati. Le differenze di carriera tra i primi cittadini risentono anche non poco della specificità delle culture politiche locali. I ritmi della carriera, tuttavia, più delle altre dimensioni, introducono una crescente uniformità tra aree geopolitiche e partiti. Rispetto ai primi anni Sessanta, si deduce una diminuzione ulteriore della durata media in carica (Spreafico calcolava 4 anni e 6 mesi per i sindaci dei comuni capoluogo; si passa a un po' più di tre anni per tutti i nostri comuni nel 1992 e a circa due anni tra i sindaci del 1993). Parallelamente, si rilevava nel 1992 un aumento della durata media della carriera: complessivamente 13 anni di esperienza elettiva, 15 anni di esperienza di partito, 2 anni in enti di secondo grado. Su questo punto l'ingresso di personale nuovo consente di segnare un po' il passo nel 1993: le relative medie si assestano rispettivamente a otto, sette e un anno. Contrariamente alle aspettative, la filière elettiva non guadagna importanza. La crisi del partito come istituzione che professionalizza l'esperienza del fare politica non sembra modificare i percorsi di carriera a favore dell'esperienza esterna al partito e di una maggiore imprenditorialità nella costruzione del cursus bonorum. Prima del 1993, le tendenze di lungo periodo della carriera politica procedevano in direzione di un allungamento dei tempi e di una moltiplicazione dell'accumulo di esperienze brevi, elettive e non; tra i sindaci eletti dopo la riforma, la prima tendenza declina decisamente, mentre la seconda pare in qualche misura tuttora in atto.

Le modalità soggettive di interpretazione della carriera, alla 
luce dei nostri dati, suggeriscono una pista di ricerca che merita ulteriori approfondimenti. Solo fra qualche anno potremo giudicare della persistenza degli elementi di rottura introdotti dalla riforma elettorale. Per il momento, si può rilevare che, tra gli eletti del 1993, la politica al livello municipale conserva la sua natura di professione ordinata, le cui regole di promozione fanno per lo più capo al partito; le pur numerose aggregazioni emergenti mascherano spesso dinamiche coalizionali e appartenenze tradizionali. $\grave{E}$ il partito che promuove la carriera e che vincola gli attori ad obiettivi definiti. Prova ne è che l'«ambizione statica» (Schlesinger 1966) a mantenere la carica è nettamente maggiore tra $\mathrm{i}$ sindaci che hanno una forte carriera intrapartitica. Inoltre, sotto molti aspetti, la continuità rispetto al passato è notevole tra le carriere dei più giovani e quelle dei veterani (in misura minore forse nel PDS).

A questa professione ordinata, tuttavia, non corrisponde un mestiere o una professionalità chiara nei suoi contorni agli occhi degli stessi attori che ne sono i protagonisti. Già prima dell'applicazione della legge 81 che voleva aprire e in parte soltanto ha aperto, come abbiamo visto, un ciclo innovativo nei processi di reclutamento dei primi cittadini, il desiderio di trasformare il proprio ruolo all'insegna della managerialità appariva forte tra $\mathrm{i}$ sindaci; e ciò sotto varie forme ${ }^{19}$. La politica non veniva più definita come la «capacità di trattare con gli uomini», ma di trattare con i regolamenti e con le risorse finanziarie offerte dalla macchina comunale in vista del raggiungimento di obiettivi concreti da realizzare in tempi brevi. Un lavoro da burocrate rispettabile, da manager nei migliori dei casi, con ambizioni dichiarate per un futuro da dirigente nella società civile $\mathrm{e}$ nel mondo dell'economia. La carriera politica sembrava essere interpretata, in una maniera sistematica e prolungata, come un'acquisizione progressiva di conoscenze spendibili anche in altri ambiti, dalla libera professione alle grosse aziende. Indipendentemente dalle innovazioni istituzionali, la tendenza a guardare sempre più fuori dagli angusti confini del municipio da parte di chi ha raggiunto l'apice del potere periferico, la dif-

\footnotetext{
${ }^{19}$ Allorché si confronti la carriera reale con la carriera ideale, grazie alla rilevazione dell'immagine idealtipica del sindaco disegnata tramite il questionario, si osserva che già nel 1992 tra le esperienze più utili per meglio ricoprire la posizione di vertice del potere municipale, la «gestione amministrativa di enti privati» veniva indicata in prima $o$ in seconda posizione (su cinque items proposti) dal $52 \%$ dei sindaci interrogati.
} 
fusione di primi cittadini che appartengono al mondo delle libere professioni, il richiamo alla validità dell'esperienza nel mondo del management suggeriscono, forse, che una nuova concezione della politica sta mettendo radici.

Riferimenti bibliografici

Barberis, C. (1973, 1978, 1983, 1988, 1993), La classe politica municipale, Milano, Angeli.

Becker, H.S. (1963), Outsiders, New York, Free Press.

Belotti, V. e M. Maraffi (1994), Ceto politico e dirigenza amministrativa nei comuni italiani, Bologna, Il Mulino.

Bettin, G. (a cura di) (1993), Classe politica e città, Padova, Cedam.

Bettin, G. e A. Magnier (1989), Il consigliere comunale, Padova, Cedam.

- (1991), Chi governa la città?, Padova, Cedam.

Black, G.S. (1970), A Theory of Professionalization in Politics, in «American Political Science Review», LXIV, 3, pp. 865-878.

- (1972), A Theory of Political Ambition: Career Choice and the Role of Structural Incentives, in «American Political Science Review», LXVI, 1, pp. 144-159.

Caciagli, M. (1991), Vita e opere di un ceto politico, in «Polis», V, 2, pp. 209-216.

Cavalli, L. (1987), Potere oligarchico e potere personale nelle democrazie moderne, in AA.VV., Leadership e democrazia, Padova, Cedam, pp. 3-54.

- (1992), Governo del leader e regime dei partiti, Bologna, Il Mulino, spec. il Cap. IV, Affermazione della «democrazia con leadership personalizzata», pp. 73-101.

- (1994), The Personalization of Leadership in Italy, Working Papers n. 2, Firenze, CIUSPO.

Cazzola, F. (1991), Periferici integrati, Bologna, Il Mulino.

Cotta, M. (1979), Classe politica e parlamento in Italia 1946-1976, Bologna, Il Mulino.

De Mucci, R. (1994), In the Byways of Italian Politics. Local Elections in the Eighties, in L. Lopez Nieto (a cura di), Local Elections in Europe, Barcelona, ICPS.

Di Virgilio, A. (1994), Elezioni locali e destrutturazione partitica. La nuova legge alla prova, in «Rivista Italiana di Scienza Politica», XXIV, 1, pp. 107-165.

Farneti, P. (1972), Problemi di ricerca e di analisi della classe politica italiana, in «Rassegna Italiana di Sociologia», XIII, 1, pp. 79-116.

Gaxie, D. (1973), Les professionnels de la politique, Paris, Seuil. 
Herzog, D. (1971), Carriera parlamentare e professionismo politico, in «Rivista Italiana di Scienza Politica», III, 4, pp. 515-544.

- (1975), Politische Karrieren, Berlin, Westdeutscher Verlag.

Hughes, E.C. (1951), Career and Office, in R. Dubin (a cura di), Human Relations in Administration. The Sociology of Organization, Englewood Cliffs, Prentice-Hall.

King, A. (1981), The Rise of the Career Politician in Britain - And Its Consequences, in «British Journal of Political Science», XI, 2, pp. 249-285.

Martinotti, G. e A. Melis (1988), Gli amministratori comunali (19751987), in «Amministrare», XVIII, 2-3.

Mastropaolo, A. (1984), Saggio sul professionismo politico, Milano, Angeli.

- (1993), Il ceto politico, Roma, NIS.

Morlino, L. e J.R. Montero (1994), Legittimità, consolidamento e crisi nell'Europa meridionale, in «Rivista Italiana di Scienza Politica», XXIV, 1, pp. 27-66.

Parisi, A. (a cura di) (1984), Luoghi e misure della politica, Bologna, Il Mulino.

Porro, N. (1993), Il cemento e la ricotta. Per una sociologia del sistema politico romano (1946-1992), Roma, Seam.

Putnam, R.D. (1976), The Comparative Study of Political Elites, Englewood Cliffs, Prentice-Hall.

Recchi, E. (1991), Il network politico dell'amministratore comunale italiano, in «Polis», V, 2, pp. 243-265.

- (1993), Tipi di network politico e tipi di sindaco, in G. Bettin, cit., pp. 227-253.

Sani, G. (1972), La professionalizzazione dei dirigenti di partito italiani, in «Rivista Italiana di Scienza Politica», II, 3, pp. 303-333.

Segatori, R. (a cura di) (1992), Istituzioni e potere politico locale, Milano, Angeli.

Schlesinger, J.A. (1966), Ambition and Politics: Political Careers in the United States, Chicago, Rand Mc Nally.

Spreafico, A. (1963), L'amministrazione e il cittadino, Milano, Comunità, al cap. IV, I sindaci dei capoluoghi di provincia, pp. 123-141.

Tarrow, S. (1979), Tra Centro e Periferia, Bologna, Il Mulino.

Tréanton, J.R. (1960), Le concept de carrière, in «Revue Française de Sociologie», I, 1, pp. 73-80. 